Z. Phon. Sprachwiss. Kommun.forsch. (ZPSK), Berlin 49(1989) 6, 729-737

Wiltrefe Herrmane (Berlin)

\title{
Zur Klassifikation der Wortarten im Koreanisehen
}

\section{Summary}

The present paper deals with a classification of the parts of speech of the liorean language giving priority to syntactico-functional and distributional criteritu, which is considered as useful for the purpose of foreign langunge teaching. Primary syntactic functions of the different word classes have been determined and the question of conversion (change of parts of specch) as well as entering of equivocal (homonymous) words or word forms, both of the same origin, into different word classes aecording to their different syntactic featuress has been discussed. Furthermore, correlations betwoen syntactically defined word classes and their morphological features have been specified.

As first step, lexical items have been divided into word classes having sentence value and not having sentenco value, respectively.

As second step, word classes lacking sentence vahu have been divided into those capablo of functioning as m independent member of sentence and those functioning mainly adjunctionally as constituent of a member of sentence, the former as to their primary or prevailing syntetetic functions (i.e. predicates, "uctunts", „circonstants") boing subdivided into vorbs, nouns und adverbs, respectively, und the latter consisting in adjectives (molifiers).

Word clusses endowed with sontence value consist of interjections and modal words.

1. Ubwohl die Klassifikation des Wortbestands konkreter Einzelsprachen ein zentrales Problem ihrer Analyso und Beschreibung bildet, wird kaum behauptet werden können, daB dieses Anliegen für irgendeine Sprache widerspruchsfrei und jedor Kritik standhaltend gelöst worden ist. Die Ursachen liegen zum einen in der Kompliziertheit des Gegenstandes als solchen, der Vielschichtigkeit des Wortbestands, sowie der Sprachstruktur überhaupt, zum anderen in der Tatsache begrändet, daß das Wort im Schnittpunkt des Interesses der lexikalischen Semantik und der Grammatik steht, dahel semantische und grammatische Kriterien zur Klassifikation des Worthestands herangezogen werden. Die grammatischen Kriterien ihrerseits sind nioht einheitlich, sondern neben morphologischen stehen syntaktisch-funktionale und distributionelle Kriterien.

Die Vorgangenheit hat - zumindest für die eingehender erforschten Sprachen hinreichond bewieson, da B mit lediglich einer der drei genannten Arten von Kriterien 1 keine brauchbare Wortartenklassifikation zustande kommt, sondern nur die komplexe

1 Auf weitere Arten, z. 13. logische Kriterien, sei hier nur beiläufig vorwiesen. Linguisten der KDVIR (so Čo.spo.v mun.pop 1960, S. 128; Ko 1987, S. 37) fassen die morphologischen Kriterien recht weit, indem sie noben der Beachtung der Besonderheiten der Formenbildung, davon ausdrücklich geschioden, auch die Beachtung der Besondorheiten der Wortbildung fordern. 
Beriicksichtigung aller drei Kriteriengruppen weiterhilft. Allerdings wird dabei nicht immer einer Gruppe von Kriterien konsequent das Primat eingeräumt, sondern nur zu oft zeigt sich, daß bei der Wortartenklassifikation eines Autors einer Wortart vorrangig semantische Kriterien zugrunde liegen, einer anderen dagegen grammatische (morphologische oder aber syntaktische) Kriterien. HwLug (1977, 102ff.) hat dies treffend für einige Wortartenklassifikationen des Deutschen dargestellt.

2. Zwar kann sich dio Erforschung des Koreanischen (Kor.) hinsichtlich Länge ihrer 'Tradition und Intensität nicht mit einer Reihe anderer Sprachen, darunter auch orientalischen messen, jedoch läßt sich auch für das Kor. die oben beschriebene unbefriedigende Situation nachweisen. Aus Platzgründen kömnen hier weder die Wortartenklassifikationen anderer Autoren für das Kor. im einzelnen vorgestellt und kritisch belenchtet noch, als Resultat einer solchen kritischen Sichtung, die strittigen Wortklassen bzw. Klassifizierungsprinzipien dargelegt werden. Die Zahl der Wortklassen schwankt zwischen 8 (bei Autoren der KJVVlR) und 12 (bei MAzUr 1954), und der Inhalt der einzelnen Wortarten im Sinne der Zuordnung verschiedener Subklassen ist auch dort, wo gleiche oder bedeutungsgleiche Bezeichnungen gebraucht werden, keineswegs einheitlich.

3. Indem wir uns nunmehr unserem eigenen Wortartensystem zuwenden, haben wir zunächst einige grundlogende Positionen zu bestimmen.

3.1. Wir teilen die Auffassung, daß ,Zielsetzung und Gesamtanlage einer Grammatik den Charakter der Klassifizierungskriterien bestimmen" (Hervorhebung Verf.) und ,auch die muttersprachliche oder fremdsprachliche Orientiorung einer Grammatik deren Wortklassensystem beeinflussen wird“ (Fı̈̈мı 1977, 41 f.), und schließen uns der Forderung Heluigs (1977, 90f.) an, der sich, и. a. NeumanN (1967, 378) „der Wortartwechsel sollte doch darauf aufmerksam machen, daß es primär dio Bedürfnisse syntaktischer Fügung sind, die Wortklassen ausprägen, da $\beta$ diese etwas mit der internen Relationsstruktur des Satzes zu tun haben "zitierend, für eine syntaktische Klassifizierung der Wortarten ausspricht: „Da nicht alle Wortarten differenzieronde Formmerkmale haben ... und auch nicht alle Wortarten cinen direkten Wirklichkeitsbezug haben ..., wohl aber jede Wortart durch ihre syntaktische Iunktion im Relationsgefüge des Satzes determinierbar sein muß, ergibt sich für uns darans die Forderung nach einer syntaktischen Klassifizierung der Wortarten“.2

Speziell in Hinblick auf den Fremdsprachenunterricht, auf eine "Grammatik für Ausländer", hält HELıı $(1968,1)$ eine syntaktische Wortartenklassifikation für angemessener, „da diese Bestimmung der Wortart von syntaktischen Funktionen her an meßbare Eigenschaften der Oberflächenstruktur anknüpft und nicht von außen an die Sprache herangetragen wird."

Dabei bedeutet das Primat eines Kriteriums nicht Negierung der anderen (morphologischen und semantischen) Kriterien, sondern diese sind im Gegenteil notwendig für die Bestimmung der "vollen Wortartcharakteristik (wie sie jede Grammatik anzustreben hat)" (Helbig 1977, S. 93) und die Subklassifikation, wo syntaktische Kriterien allein nicht ausreichen. In Hinblick auf den wechselseitigen Zusammenhang

2Vgl. auch Helmig/Buscha 1987, S. 19; ferner Meier 1979, S. 32, 35, der fordert, dio Grundwortarten aus der Satzgliedanalyse funktional abzuleiten bzw. Wortklassen in erster Linie funktionalgrammatisch zu erfassen. 
dieser drei Arten von Kriterien - bei Primat der grammatischen Kriterien - wird man sich dem Gedanken nicht versehließen können, daß die begrifflich-kategoriale (iuhaltliche) Wortklassenprägung - als semantisches Kriterium - in gewissem Grade aus der syntaktisch-morphologischen resultiert, ein IReflex der letzteren ist (vgl. Flämig 1977, 43; Moskal'skaja 1977, 144; Meleir 1979, 27). Zum anderen ist zu bedenken, daß3 ,zahlreiche syntaktische Funktionen der Wörter ihren Niederschlag in einem entsprechenden Ausbau ihres Flexionsparadigmas gefunden haben" und folglich die morphologischen Besonderheiten einzelner Wortklassen ,auch die Eignung der Wörter, bestimmte Funktionen im Satz zu übernehmen", anzeigen (FL̈̈mG 1977, 44), was auf den (mitunter sehr engen) Zusammenhang zwischen morphologischen und syntalitischen Klassifizierungskriterien und die Möglichkeit der (u. U. völligen) gegenseitigen Uberdeckung morphologisch bzw. syntaktisch bestimmter Wortk]nssen hindeutet.

3.2. Die Klassifikation des Wortbestands setzt die Definition des Wortes voraus. Sicher ist dem zuzustimmen, daß, ,es erhebliche Schwierigkeiten (bereitet), das Wort ganzheitlich zu erfassen" (Fı̈̈Mg 1977, 39), und „daß eine fïr alle Sprachen der Erde gïltige Definition des 'Wortes' nicht möglich und auch nicht sinnvoll ist, da 3 aber andererseits in jeder Sprache Einheiten existieren, die die Grundelemente der Lexik der jeweiligen Sprache ausmachen, also Wörter sind, die jedoch in Utbereinstimmung mit dem jeweiligen System der Sprache definiert werden miissen “ (MYIER 1981, 51). Mit Flämig (1977, 40) wollen wir daher „vom Wort als Einheit der linguistischen Praxis ausgehen“ und Wörter ,als kleinste relativ selbständige, d. h. potentiell isolierbare sprachliche Bedeutungsträger; kombinierbare, in Wortgruppen/ Sätzen austauschbare, wmstellbare und durch Ginschub voneinander trennbare Einheiten mit spezifischer morphematischer Struktur" auffassen.

1)as orthographische Kriterium, hier speziell Festlegungen hinsichtlich der Getrenntund Zusammenschreibung, schließen wir als willkürliche (xrößße aus (vgl. Nkifn 1961, 295). Allerdings stehen hiermit die Frage des syntagmatischen Worts, die Unterscheidung zwischen Wortgruppe und Kompositum bzw. der Ubergang von Wortgruppen zu Komposita im Zusammenhang. Dennoch ist es für unser Anliegen ohne Belang, ob \%. B. purk.un $k i$ 'rote Fahne' als determinatives Syntagma purk.un 'rot' + ki 'Fahne' oder als Ncologismus aufgefa ßt und daher entweder getrennt-oder zusammengeschriehen wird. Als Beispiel für ein prädikatives Syntagma sei der l'hraseologismus krp.ur mvk.ta 'sich fürchten' (wörtl. 'Furcht essen') angefïhrt. Wegfall der Akkusativendung -ur in dieser Objekt-Prädikat-Wortgr'up)e( $\rightarrow k n p . m n k . t a)$ kann als Indikator für Ioxikalisier'ung aufgefalt werden, dio auch hier mit der orthographischen Konsequenz der Zusammenschreibung verbunden ist. Da im Kor. für die syntaktische Funktion des Syntagmas (als komplexer Komponente eines Satzes) obenso wio die eines Kompositums stots das in hinterer Position erscheinende Lexem entscheidend ist, impliziert der Ubergang der Wortgruppe zum Kompositum keine Veränderung in dieser Hinsicht und sollte die Frage Wortgruppe/Kompositum bzw. Getrennt-/Zusammenschrejbung mindestens für eine syntaktisch orientierte Wortartenklassifikation vernachlässigbar sein.

Die Definition des Wortes involviert ferner die Verständigung darüber, welchen oder welcher Art Morphemen nicht der Status eines Worts zuerkannt werden kann. Auch hier sollten orthographische Festlegungen mit äuBerster Vorsicht berücksichtigt 
werden. Einige Autoren billigen den Verben (Prädikativen) Flexion zu, während sie diese den Nomina (Substantiven, Pronomina, Numeralia) absprechen 3, was impliziert, da B. sie den an Nomina antretenden gebundenen Morphemen den Status von Postpositionen, Partikeln, Hilfswörtel’n, Relationswörtern u. dgl. zusprechen. Wir können dieser Auffussung nicht folgen und behandeln daher - unabhängig von der Fragestellung, ob das Kor. eine agglutinierende oder flektierende Sprache oder aber eine agglutinierende Sprache mit Elementen flektierenden Typs ist - neben den Verbalendungen auch die syntaktischen Endungen (Kasusendungen) der Nomina und die sog. modifizierenden Endungen' nicht als eine oder mehrere Wortklassen, sondern als unfreie Morpheme (Grammateme). In Úbereinstimmung mit den meisten anderen Antoren und im Unterschied zu den Linguisten der KDVR erkennen wir dagegen die Existenz einos Kopulaverbs 'sein' mit der' Wurzel $i$ - an.5

3.3. Bei einer vorrangig syntaktischen Wortartenbestimmung kommt der Zuweisung der primären syntaktischen Funktion(en) besondere Bedeutungzu, und dabei ist auch die Frage des Wortartwechsels von Interesse. Ist beispielsweise die Bildung der attributiven Form oder der Adverbialform eines qualitativen Verbs, z. P. noph.ta 'hoch sein'>noph.uen 'ein(e) hohe(r)' usw./noph.i 'hoch' (Adverbialform), gleichbedentend mit einem Clbertritt in die Wortklasse Adjektivwörter (Attributiva) bzw. Adverbien? Ahnliche C̈berlegungen gelten den Adjektiven auf -čbl, die eine Adverbialform auf -ezk.u.ro (durch Anfiigen der Instrumentalendung) bilden. Wir entscheiden hier so, daß damn kein Wortartwechsel vorliegt, wenn Ableitungen regulär und zugleich produktiv gebildet sind, l. h. ihre Bildung von dem, der die Regel kennt, uneingeschränkt nachvollzogen werden kann. In dieser Herangehensweise ̈̈uBert sich unser Bestreben, den Bolangen des Fremdsprachenunterrichts Rechnung zu tragen (vgl, hierzu Heubla/Buscina 1980, 18). I)as veranlabt uns, z. B. im Falle der Ableitung von Adjektiven auf - ¿̋j aus sinokor. Substantiven auf Wortartwechsel zu erkemen, nicht aber im Falle der Ableitung der Adverbialform von diesen Adjektiven durch Anfügen von -u.ro. Die Ableitung der attributiven Form(en) qualitativer Verben, die ja mit Adjektivwörtern, darunter den Adjektiven auf -čnk, funktionsgleich sind, verläbt dagegen nicht den Bereich der Formbildung, zumal attributive Verbalformen generell Tempusbedentung (relative 'T'empora) einschließen.

Problematisch erscheint die Einordnung der Adverhialform(en) qualitativer Verben. Während die regulär auf -ke abgeleitete Form gemäls Obigem zweifelsfrei in den Bereich der Formenbildung gehört (kein Wortartwechsel), bedarf die Hntscheidung hinsichtlich der Adverbialform nuf $-i /-h i$ weiterer Uberlegungen, da sie erstens nicht von allen Elementen dieser Wortklasse und zweitens zum Teil unregelmäßig gebildet wird.

Diskutierenswert erscheint ferner die Behandlung der Verbalsubstantive (Dever-

3 Die Flexionslosigkeit weiterer Wortarten, so der Adverbien und Attributiva, ist unum. stritten und hier nicht von Interesse.

"Modifizierendo oder "Hilfs"endungen entsprochen semantisch etwa den Partikeln des Deutschen und werden an Wortstämme von Nomina und Adverbien, an Kasusformon von Nomina so wio auch an Verbformen angefügt. Vorrangig sind aie aber wohl der Formenbildung der Nomina zuzuordnen.

5 Die Linguisten der KDVR (so u. a. Ko 1987, S. 165; vgl. ulch Čo.svn.v mun.ppp 1960, S. 181) vertroten dio Auffassung, daß es sich bei diesem $i$ nicht un eine Wortwurzel, sondern un oin verbalisierendes Affix, also ein Wortartwechselmorphem handelt. 
bativa, nominalisierte Verbformen) auf $-m$ und $-k i$. Morphologisch verhalten sie sich wie Substantive: Antreten von Kasusendungen und modifizierenden Endungen (s. Fu Bn. 4). Syntaktisch dagegen schwanken sie - unter funktionalem wie auch distributionellem Aspekt - zwischen Substantiv und Verb: (1) Mitunter verhalten sie sich uneingeschränkt wie ein Substantiv, d. h. sie bilden Wortgruppen mit Attributen, z. B. tii.toy.liay.ui ju.ju.han hum.vum u.e 'auf der trägen Strömung des Tüdong-Flısses' (al)geleitet von der Verbwurzel hu.rue- ‘fließen, strömen'). (2) Jn anderen Fällen verhalten sie sich als Prädikat eines untergeordneten 'Jeilsatzes wie ein Verb, d. h. sie haben Aktanten und freie Angaben bei sich, z. B. lii.loy.kay.un ju.ju.hi hu.vum.e.to pur.ku.hu.ku 'obwohl (ungeachtet dessen da B) der 'Tädong-FluB triige duhinströmt'. Im Fall (1) sollte auf Wortartwechsel (Ableitung eines Jeverbativuns) erkamnt werden, im Fall (2) dngegen nicht, sondern auf Bildung eines formal-funktionalen Verbalnomens. Dafür spricht anch, daß grammatische Kategorien des Verbs wie subjektbezogene Höflichkeit und Tempus im Fall (2) durch die entsprechenden Suffixe ausgedriickt werden können, dagegen im Fall (1) nicht präsent sind. Forner kann im Fall (1) begrifflich-kategoriale Umprägung (auch wenn der (Gedanke nicht fallengelassen wird, dals diese ein Reflex grammatischer 'Tatbestände ist) und daher Wortartwechsel angenommen werden, während im Fall (2) das formal-funktionale Verbalnomen die begrifflich-kategoriale Prägung des Verbs behält, also auch aus dieser Sicht kein Wortartwechsel stattfindet.

J)ie zuletzt behandelte kischeinung wirft die Frage nach der Behandlung von Homonymen auf (vgl. 3.4.). Auch sollte del Gedanke von Cindmitonova (1977), Wortklassen in Zentrum und periphere Bereiche aufzuteilen, berücksichtigt werden: Die Verbalsubstantive hätten dam ihren Platz zweifellos an der Peripherie einer Wortart Nomen bow, einer Subklasse Substantiv.

Auf jeden Fall muß in Rechmung gestellt werden, daß eine bestimmte syntaktische Position besetzt. werden kann (a) von cinem unabgeleiteten oder durch ein derivationelles Morphem - ggf. wnter Wortartwechsel - abgeleitetes Wort, das primäl dio hetreffende Funktion lat, oder (b) von einem Wort, dessen primäre Funktion eine andere ist, das aber vermittelt durch ein Grammatem (Formbildungsmorphem) oder Hilfswort (Funktionswort) sekundär diese Funktion ïhernimmt. ("/ur Behandlung von Wortgruppen vgl. 3.2.)

3.4. Wie nich oben am Beispiel der Verbalsubstantive zeigte, ist die Zuordnung homonymer Wörter oder Wortformen zu unterschiedlichen Wortklassen ins Auge zu fassen, wem sie unterschiedliches syntaktisches Verhalten zeigen. HuLisa/ Brscua verfahren so, wem sie \%. B. der, die, des mnter Artikelwörtern, Relativpronomina und (umgangssprachlich) Jemonstrativpromomina einordnen (1987, 236,

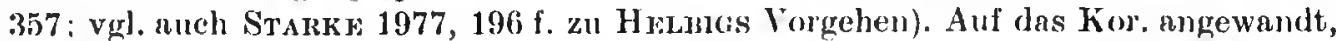
berechtigt dies, gestützt auch durch morphologische Unterschiede, u. a. die Numernlien und die - allgemein formuliert - deiktischen Wörter $i, h u$, čn unter verschiedenen Wortarten einzuordnen, wie dies schon von Cü̈ HJòn P̈̈ (1961, 565ff.) vorgeschlagen wurde (vgl. anch Henmane 1985, 157).6

" Dal. Wortpanre wie z. B. kiph.i 'tief' (Adverbinlform) und kiph.i 'Tiefe' (boide von der Verlowurzel kiph - 'tief sein' abgeleitet) Homonyme sind, wird kam nnzuzweifeln sein. In den anleren hier aufgefïhrten Fällen, vor allem in bezng nuf Ableitungen des 'Typs liu.rum" (vgl. 3.3.), wïre zu diskutieren, olo es sich nicht un Polysemie handelt. Da es hier 


\section{Klassifizierungsvorschlag}

Wir unterteilen den Wortschatz zunächst in satzwertige und nicht-satzwertige Kinheiten.

4.1. Wir betrachten zuerst die nicht-satzwertigen lexikalischen Einheiten. Sie lassen sich - nach den primär iibernommenen syntaktischen Funktionen - in satzgliedfähige und gliedteilfähige untergliedern, davon die ersteren in prädikatfähige und nicht-prädikatfähige, darunter die letztgenannten in aktantenfähige und (iiberwiegend) angabenfähige. Wir gelangen auf diese Weise fiir den Bereich der nicht-sat\%wertigen lexikalischen Einheiten zu den folgenden vier Haupt-Wortklassen:

\subsubsection{Verben (Prädikative)}

Ihre primäre Funktion ist die Besetzung des Prädikats. Der verbozentrischen Sprachauffassung folgend und eingedenk der Tatsache, daB das Satzminimum im Kor. das Prädikat ist 7 - alle iibrigen Satzglieder können weggelassen werden, wenn dic kommunikative Situation es gestattet - setzen wir diese Haupt-Wortklasse an die erste Stelle.

\subsubsection{Nomina (Substantivwörter)}

Ihre wesentliche primäre Funktion ist die Besetzung der Leerstellen (Aktanten, Mitspieler) des Verbs im Sinne der Valenztheorie: Subjekt, Objekt(e), Adverbialergänzungen - aber auch freie Adverbialangaben, da diese sich äuBerlich häufig nicht von Adverbialergänzungen unterscheiden. Weiterhin wird auch die Attribut-(Gliedteil-)funktion zu den primären syntaktischen Funktionen der Nomina zu rechnen sein.

Verben und Nomina bilden damit die Grundkonstituenten des Satzes, sind daher als (Hrund-Wortklassen anzusehen. Die nach syntaktischen Kriterien vorgenommene Differenzierung dieser zwei Grund-Wortklassen findet ihre Entsprechung in den morphologischen Merkmalen: Das Formenparadigma, das zugleich auch dic \%uordnung unterschiedlicher grammatischer Kategorien gestattet - partnerbezogene und subjektbezogene Höflichkeit, Modus, Modalität, Tempus u. a. bei den Verben; Kasus, (fakultativer) Plural u. a. bei den Nomina - hebt sie voneinander al), bzw. dic syntaktische Funktion wird durch die Wortform bestimmt und umgekehrt.

\subsubsection{Adverbien (Verbbegleiter)}

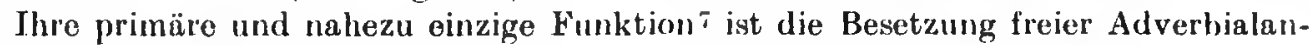
gaben. Als valenzabhängiges Adverbial (Adverbialergänzung) treten sie seltener als im Deutschen in Erscheinung, da ein großer Teil der deutschen Adverbien mit ProwortCharakter kor. Aquivalente in Gestalt von Nominalformen (besonders Pronominalformen) hat und eine Reihe anderer Adverbien des Deutschen Komponenten kor. Verb-Verh-Komposita entspricht. Inwieweit auch im Kor. bestimmten Gruppen von Adverbien Gliedteilfunktion zukommt (vgl. Conran 1985, 15; Fl,̈̈uG 1981, 689f.) besonders in Beispielen wie Iru.rnh.ke a.rum.tap.ta 'so schön sein', a.ču coh.uın

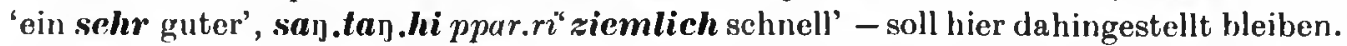

aber um die Zuordnung äquivoker Einheiten zu unterschiedlichen grammatisch be. stimmten Wortklassen geht, erscheint es angemessener, von (grammatischer) Homony nie zu sprechen (vgl. Allgemeine Sprachwissenschaft, Bd. II, 1975, S. 373).

7 Dies gilt zumindest für die Schriftsprache (Literatursprache, Standardsprache). Und gerade sie bildet den Hauptvermittlungsgegenstand des Fremdsprachenunterrichts. 
Zu überdenken wäre diese Frage in Verbindung mit der Definition des Satzminimums nicht nur für den Satz als Ganzes bzw. den Hauptsatz, sondern auch für Nebensätze.

Adverbien gelten im Unterschied zu Verben und Nomina generell als unflektiert. Es darf jedoch nicht übersehen werden, da $B$ auch an Adverbien modifizierende Endungen antreten können (s. Fu Bn. 4). Die Adverbien verhalten sich in dieser Hinsicht ganz so wie als Adverbial fungierende Kasusformen der Nomina (einschließlich Verbalnomina).

4.1.4. Adjektivwörter (Nomenbegleiter)

Sie haben überwiegend nur Gliedteilfunktion, d. h. sie treten als Attribut auf. Lediglich die von uns hierunter als eine Subklasse eingeordneten auf -čpk abgeleiteten Adjektive sind in der Lage, eine Adverbialform zu bilden sowie, vermittelt durch die Kopula i.ta 'sein', Prädikatsfunktion auszuüben. Bis auf die letztgenannto Subklasse sind die Adjektivwörter durch Fehlen jeglicher Formenbildung gekennzeichnet: Es treten auch keine modifizierenden Endungen an.

Wenn wir oben Verb und Nomen als Grund-Wortklassen bezeichnet haben, geschah dies auch mit Rücksicht darauf, daß Adverbien und Adjektivwörter primär Klassen von Beiwörtern bilden: die Adverbien in erster Linie als Verbbegleiter und die Adjektivwörter als Nomenbegleiter.

Betrachten wir die Fähigkeit der vier Haupt-Wortklassen, weitero Satzgliedfunktionen außer den jeweils als primär angesehenen zu übernehmen, sind Verb und Nomen den anderen Wortklassen deutlich überlegen. ${ }^{8}$ Regulär abgeleitete Verbformen treten häufig als Attribute oder Adverbiale auf und können auch die Positionen von Subjekt und Objekt(en) besetzen. Nomina besetzen neben Aktanten-, Adverbial- und Attributpositionen auch die Position des Prädikats, wobei der Kopula i.ta eine Mittlerrolle als Hilfsverb (Funktionswort) zukommt. Adverbien und Adjektivwörter weisen dagegen keine derart breite Palette syntaktischer Einsatzmöglichkeiten auf. Teils sind ihre sekundären Funktionen umstritten, oder sio gelten nur für Teilklassen.

4.2. Zu den satzwertigen Wortschatzeinheiten werden gewöhnlich die Interjektionen und die Modalwörter gerechnet. Der Unterschied zwischen ihnen besteht neben semantischen Gesichtspunkten - unter grammatischem Aspekt allgemein gesagt darin, daß Modalwörter oberflächensyntaktisch das Verhalten von Adverbien, also eines Satzglieds zeigen (daher auch von vielen Autoren als eine Subklnsse der Adverhien gefa (3t werden), tiefensyntaktisch, d. h. in einer zugrunde liegenden abstrakten syntaktischen Struktur jedoch Satzwert offenbaren, während sich Interjektionen auch oberflüchensyntaktisch als satzwertige Einheiten erweisen, keine Satzgliedfunktion haben (vgl. Helbig 1977, 106, 110f.; Helbia/Buscira 1987, 503ff.; Moskal'skasa 1977, 140). Interjektionen sind also nicht Wörter im Sinne von Bausteinen von Sätzen und bilden daher nach Ansicht wohl der meisten Linguisten ,keine Wortklasse im eigentlichen Sinne" (FLÄMı 1981, 491). Es ist jedoch zu bedenken, daß auch die Interjektionen als isolierbare Elemente des Wortschatzes in Wörterbiicher aufgenommen werden. Deshalb plädieren wir unter praktischem Aspekt dafür, ihnen auch in der Wortartenklassifikation einen, wenn auch deutlich abgehobenen Platz einzuräımen.

4.3. Abschließend soll kurz auf die Subklassifikation der oben bestimmten Haupt-Wortklassen eingegangen werden. Diese bildet u. E. einen wichtigen Prüfstein

${ }^{8}$ Vgl. Fußn. 7. 
für die Adäquatheit der vorgeschlagenen Wortartenklassifikation, differieren hier doch die Ansichten z. T. erheblich. Platzgründe erlauben uns nur ein oberflächliches Eingehen, wobei wir uns auf die nicht-satzwertigen Wortklassen beschränken.

4.3.1. Als Subklassen der Verben können auf der Grundlage semantischer Merkmale, die ihre Widerspiegelung auch in Besonderheiten des Formenbildungsparadigmas finden, jrozessive Verben, qualitative Verben, Existentialverben und Kopulaverben unterschieden werden.

4.3.2. Subklassen der Nomina sind die Substantive (einschließlich der Numeralklassifikatoren und der defektiven Substantive bzw. Hilfsnomina), die Pronomina und die substantivisch gebrauchten Numeralia. Dabei grenzen wir die Klassen der Pronomina und Numeralia auf solche Elemente ein, die unter distributiven Gesichtspunkten anstelle von Substantiven in gegebene Substitutionsrahmen einsetzbar sind. Alle Subklassen der Nomina haben dasselbe Formenbildungsparadigma, unterscheiden sich aber in der inhaltlichen Prägung.

4.3.3. Die Adverbien bieten zweifellos das heterogenste Bild. Als problematische Subklassen seien hier nur die Modalwörter (vgl. 4.2.), die Konjunktionaladverbien und die Onomatopotika genannt. Die Onomatopoetika als gesonderte Subklasse der Adverbien zu behandeln - manche Autoren setzen sie sogar als eigene Wortklasse neben den Adverbien an (vgl. Ko 1987, 52) - erscheint insofern bedenklich, als dann gleichberechtigt auch eine analoge Subklasse bei den Verben einzurichten wäre. Denn von einer Vielzahl von Onomatopoctika werden Verben abgeleitet.

4.3.4. Als Subklassen der Adjektivwörter betrachten wir die Adjektive des Typs sï 'neu', die z. T. wie Präfixe wisken und deren Abgrenzung vou den eigentlichen Präfixen daher auch umstritten ist (vgl. Ko 1987, 173ff.; Co.son.p mun.ppp 1960, 452; C̆ı̈ 1961, 648), die schon melıfach erwähnten, mit dem Ableitungsmorphem - $c v k$, seltener der Genitivendung - $u i$ von sinokor. Substantiven abgeleiteten Adjektive, Einheiten mit Prowort-Charakter (hinweisend/verweisend, fragend, indefinit, verallgemeinernd), soweit sie in gegebenen Substitutionsrahmen die Stelle eines Attributs besetzen können, und attributiv gebrauchte Numeralia. Bei den zwei letztgenannten Subklassen ergeben sich Uberschmeidıngen mit den entsprechenden Subklassen der Nomina (Pronomina und Numeralia), die wir gemäß 3.4. unter dem Gesichtspunkt der Homonymie Jösen.

Als Besonderheit des Kor. kam gelten, daß bei sämtlichen obigen Haupt-Wortarten, auch bei den Verben, relativ geschlossene Klassen von Prowörtern existieren (vgl. Herrmann 1985). Eine weitere Besonderheit sehen wir darin, daß Funktions-oder Hilfswörter nicht als eigenständige Wortarten, analog etwa den deutschen Präpositionen und Konjunktionen, herausgearbeitet werden können, sondern entsprechend ihren distributionellen und morphologischen Merkmalen als relativ geschlossene Subklassen der Verben und Nomina (sofern man Konjunktionaladverbien und Modalwörter ebenfalls zu den Funktionswörter'n rechmet, auch als Subklassen der Adverbien) zu gelten haben. ${ }^{0}$

${ }^{9}$ Faßs man die Nodalwörter als selbstïndigo Wortart, wio wir es hier vorgeschlagen haben, wären sie folglich die einzige eigenständige Klasse von Funktionswörtern (nicht Subklasse ciner anderen Wortart). Dies sollte Anlaß3 zu weiteren Untersuchungen sein, ob Modalwörter als selbständige Wortart unter den spezifischen Gegebenheiten des Kor. dem Primat syntaktischer Kriterien letztendlich gerecht werden. 
Wir stimmen mit Helbig $(1977,104)$ darin überein, daß für Belange des Fremdsprachenunterrichts eine möglichst explizite linguistische Beschreibung anzustreben ist, was bei den Wortarten die Aufgliederung in möglichst viele Sub- und Teilklassen verlangt. Andererseits erfordert gerade eine solche explizite Beschreibung ein hohes Maß an sprachlicher Kompetenz, letztendlich die Kompetenz des Muttersprachlers. Denn nur dieser kann bei den hierzu notwendigen vielfältigen Substitutionsproben (einschließlich Weglassung und Hinzufügung), l'ermutationsproben und Transformationsproben in letzter Instanz grammatische Sätze von ungramnatischen trennen.

\section{Literatur}

Allgemeine Spruchwissenschaft (1975): Von einem Autorenkollektiv unter Leitung von B. A. SERIBRENNIKov, Bd. II. Berlin.

Chantanova, 1.J.(1977): Zur Frage von Zentrum and Peripherie einer Worlart in Deutschen, in: Hetbig, G. (H rsg.), Beiträge zur Klnssifizierung der Wortarten, Leipzig, S. $28-38$.

С̆ö Juòn $P_{\mathrm{A}}$ (1961): U.ri mar.pon, 3. überarb. Aufl., Nòul.

Conrad, 12. (Hrgg.) (1985): Lexikon sprachwissenschaftlicher Termini, I,eipzig.

Co.svn.v mun.pop (1960): (Autorenkollektiv), Bł. I, l.hjongjang 1960.

FLÄмIG, W. (1977): Zur grammatischen Klassifizierung des W'ortbestandes im Deutschen, in: Helbig, G. (Hrsg.), Beiträge zur Klassifizierung der I'ortarten, Leipzig. న. 39-52.

- (1981), in: Grundzüge einer deutsrhen Grammatik, (Autorenkollektiv), Berlin.

Helbia, G. (1968): Zum Problem der IIortarten in einer deutschen Grammatik für Ausländer, in: Deutsch als Fremdsprache $51, \mathrm{~S} .1-18$.

- (1977): "u einigen Problemen der W'ortarthlassifizierung im Deutsehen, in: 7seiträge zur Klassifizierung der Wortarten, Leipzig, s. 90-118.

Heimig, G., 13uscha, J. (1980): Deutsche Grammatik - Ein Handbuch für den Ausländeruntervicht, 6. Aull. Leipzig, (10. Aufl., 1987).

Hermana, W. (1985): Zum System der Prowijter im Koreanischen, in: ZL'Ś, 38, 2, S. $155-165$.

Ko Sin Suk (1987): Čo.svn.p ri.ron mun.ppp (phum.sh.ron), Phjongjnng.

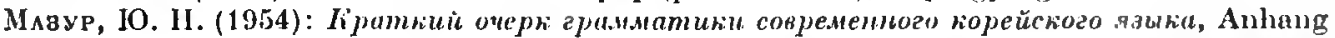
zu: "улскко-корейский словарь, Москиа.

Menwe, G. F. (1961): Kriteria für die Defintion rles Wortes, in: ZPSK, 14, 4, S. 294-297.

- (1979): Wortklassen als Busis jeder Grammatiktheorie (\%ur Methodologie der Wortklassifikation in asiatischen und europäischen Spruchen), in: ZPSK 32, 1, \$. 24-35.

- (1981): Wortprobleme in asiatischen Sprachen, in: ZPSK, 34, 1, S. 51-58.

Moskal'skaja, O. (1977): Zur Wortartentheorie, in: HeLbig, G. (Hrag.), Beiträge zur Klassifizierung der Wortarten, Leipzig, S. 138-147.

Neumann, W. (1967): Rezension zu W.Jung, Grommatik der deutschen sprurhe, in: ZPSK, 20, 4, S. 371-380.

Stanke, G. (1977): Zur Abgrenzung und Subllnssifizierung der Adjektive und Adverbien, in: HeLuG, G. (Hrsg.), Beiträge zur Klassifizierung ler Wortarten, Leipzig, \$. 190-203.

Dr. Wufreded Herrmavs, Humboldt-Universität zu Berlin, Sektion Asienwissenschaften, Abt. Korea 